\title{
Experimental Infection of Enterotoxemic Escherichia coli Associated with Porcine Edema Disease and its Pathologic Characteristics in the Intestine
}

\author{
Takamitsu TSUKAHARA ${ }^{1,2)}$, Nobuo NAKANISHI ${ }^{3)}$, Keizo NAKAYAMA ${ }^{2)}$, Noritaka MATSUBARA ${ }^{4)}$ and \\ Kazunari USHIDA ${ }^{1)}$ \\ ${ }^{1)}$ Laboratory of Animal Science, Kyoto Prefectural University, Shimogamo, Kyoto 606-8522, 2) Kyoto Institute of Nutrition and Pathology, \\ 577 Omote, Kyoto 612-8219, ${ }^{3)}$ KYODOKEN Institute, 585 Shimoitabashi, Kyoto 612-8073 and ${ }^{4)}$ Combi Corporation, Saitama 338-0832, \\ Japan
}

(Received 13 May 2005/Accepted 7 July 2005)

ABSTRACT. Edema disease (ED) has become frequent in Japan, but no effective method for experimental infection has been developed. We report here the use of a capsule that resistant against gastric digestion to induce the ED in piglets. Four 21-day-old piglets were used. Shiga toxin 2e-producing Escherichia coli (STEC) cell pellet was encapsulated and administered orally. Two pigs received 1.0 $\times 10^{10} \mathrm{CFU}$ for two days, and the others received $3.9 \times 10^{8} \mathrm{CFU}$ for three days. The high-dose group caused the typical clinical ED signs (palpebral edema or neurologic impairment). Eosinophil infiltration, swollen lymphoid follicles, and edema were observed in the ileum. The kidney had the thrombus in the glomerulus.

KEY WORDS: edema disease, eosinophil infiltration, experimental infection.

J. Vet. Med. Sci. 67(11): 1167-1171, 2005

Edema disease (ED), a sporadic disease of weaned pigs caused by host-adapted Escherichia coli, produces Shiga toxin 2e (Stx 2e) [2]. Stx 2e-producing E. coli (STEC) colonizes the lower small intestine in the pigs by F18 fimbriae, produces Stx 2e locally, and develops ED [2, 4]. Clinical symptoms associated with ED include palpebral edema, neurological impairment, lateral recumbence, and sudden death $[2,5,14]$. Nowadays, the occurrence of ED has been increasing rapidly in Japan $[25,26]$. However, the etiology of this disease remains unknown, and its control is difficult [22]. Antimicrobial therapy is used as a major curative treatment for piglets with ED. However, the use of antimicrobials has resulted in the development of antimicrobialresistant STEC on Japanese farms [8, 22]. Furthermore, the use of some antimicrobials, such as ampicillin and fosfomycin, is a possible cause for the aggravation of ED symptoms [23]. Accordingly, the use of materials other than antimicrobials is requested for the prevention or treatment of ED, most particularly on farms where antimicrobial-resistant STEC has been identified. Experimental STEC infection is an essential tool to evaluate the potential of the materials that could prevent the ED. However, the experimental oral infection led to the development of clinical symptoms in $30 \%$ of STEC-infected piglets $[5,7,14]$. In this report, we propose an improved technique for oral infection using capsules that carry STEC down to the small intestine to enable the development of the clinical signs of ED in the weaned piglets. In addition, some histological characteristics of the intestinal mucosa during the development of ED are also shown in the present report, because eosinophils infiltration was suggested to be a common pathological characteristic of the ED piglets $[13,17]$.

Four ( 2 males and 2 females) 21-day-old crossbred (Landrace $\times$ Large white $\times$ Duroc) piglets weighing approximately $5 \mathrm{~kg}$ were purchased from a commercial pig farm.
The animals were healthy and had not received any antimicrobials prior to the study. The animals were housed in pens at KYODOKEN Institute, Kyoto. The temperature was kept with brooders at $30^{\circ} \mathrm{C}$ (determined on the floor just under the brooder). The animals were fed a commercial diet for weaning piglets (SDS No.1; Nippon Formula Feed, Yokohama, Japan). The diet was free from intestinal microbiota modifiers, such as antimicrobials and probiotics. The nutrient composition of this diet was the same as that described by Tsukahara et al. [20]. The diet and water were given $a d$ libitum throughout the study. The experimental animals were handled in accordance with the guideline for animal studies of KYODOKEN Institute. E. coli strain 1362-1 serogroup O139 was kindly obtained from Dr. M. Sueyoshi, Miyazaki University, Miyazaki, Japan, and was used to induce the edema disease in this study. This strain was originally isolated from a dead piglet that had lived for 40 days on a commercial pig farm. Strain 1362-1 is hemolytic and produces Stx 2e, a heat-stable enterotoxin, and the F18 fimbriae, which mediate colonization in the intestine of weaning piglets [4]. This STEC was cultured on a TS broth medium (10 mL; Difco, Detroit, MI, U.S.A.) with an aerobic condition at $37^{\circ} \mathrm{C}$. After 4 to $8 \mathrm{hr}$ incubation, the cells were harvested by centrifugation $(3,000 \mathrm{rpm}, 15 \mathrm{~min})$. The volume of the bacterial pellets was approximately $0.5 \mathrm{ml}$. Cell pellets were poured in a capsule (Sansyoiyaku Co., Ltd., Shizuoka, Japan), that was resistant to gastric digestion, but it was readily digested in the small intestine. The cell pellet capsules were prepared just before use.

The experimental procedure was principally the same as that described by Bosworth et al. [5]. Briefly, 4 pigs were divided into 2 groups of 1 male and 1 female, and each group was housed in a pen with a brooder. After 4 days of adaptation to the diet and the environmental conditions, one group of pigs received a capsule orally, which contained 1.0 
$\times 10^{10} \mathrm{CFU}$ of STEC (high-dose group). The other group of pigs received a capsule orally, which contained $3.9 \times 10^{8}$ CFU of STEC (low-dose group). The plan was to give the capsules for 3 consecutive days ( $\mathrm{d} 0, \mathrm{~d} 1$, and $\mathrm{d} 2$ ). However, 2 pigs which received a high dose of STEC showed severe diarrhea on $\mathrm{d} 1$; therefore, the STEC challenge on $\mathrm{d} 1$ was only canceled (administered at $\mathrm{d} 0$ and d2). The clinical symptoms of edema disease, such as palpebral edema, neurologic impairment, lateral recumbency, or death, loss of appetite and vitality, respiratory difficulties, and diarrhea were checked daily. A rectal swab was collected on $\mathrm{d} 0, \mathrm{~d} 1$, $\mathrm{d} 7$, and $\mathrm{d} 14$. Body weight was also measured on $\mathrm{d} 0, \mathrm{~d} 7$, and d14. On d7, a female piglet in each group was euthanized by exsanguination under general anesthesia with an intramuscular injection of Ketamine hydrochloride (Veterinary Ketalar 50; Sankyo, Tokyo, Japan) and Azaperone (Stresnil; Sankyo). On d14, a remaining male piglet in each group was euthanized in the same manner. The each segment of intestine was longitudinally incised, and the luminal contents were carefully collected from the jejunum, ileum, and cecum. The mid- and end- segments of the ileum and the mid-segment of the gyri centripetales were fixed into a $10 \%$ $(\mathrm{v} / \mathrm{v})$-neutralized buffered formalin solution. Other organs and tissues were also collected and fixed when they appeared abnormal.

The classical bacteriological methods and PCR were used in this study to detect the challenged STEC bacterium in the samples of rectal swabs and intestinal digesta [19]. Briefly, rectal swabs and digesta of the jejunum, the ileum, and the cecum were subjected to serial ten-fold dilution, and diluted solutions were inoculated into DHL agar plates (Nissui, Tokyo, Japan) and incubated for 18 or $24 \mathrm{hr}$ at $37^{\circ} \mathrm{C}$. E. coli colonies (pink in color) were picked up and streaked onto Heart Infusion agar plates (Difco) containing sheep defibrinated blood $[5 \%(\mathrm{v} / \mathrm{v})]$ and further incubated for 18 or $24 \mathrm{hr}$ at $37^{\circ} \mathrm{C}$. Hemolytic colonies consisting of rod-shaped bacteria that were stained Gram-negative were identified by Api 20E (Nihon bioMerieux, Tokyo, Japan). Three representative hemolytic $E$. coli colonies with the highest dilution rate on a DHL agar plate were adopted to detect the Stx 2e genes by the PCR method $[3,11]$ for final confirmation. A lung with a tubercle and pus in the thoracic cavity (highdose group) were also examined. Histological studies were basically the same as those described elsewhere [21]. Briefly, the fixed intestine samples were further cut transversely into tissue block with an approximate length of 10 $\mathrm{mm}$. Three-mm-thick cross sections were prepared and stained with hematoxylin and eosin (HE) or Luna for a microscopic examination. The abnormal organs were also examined for microscopic analyses with HE stain.

The clinical sign of edema disease (palpebral edema or neurologic impairment) was quite obvious in the high-dose group from $\mathrm{d} 2$ to autopsy (d7 or $\mathrm{d} 14$, respectively) (Table 1). On the other hand, the clinical sign of the low-dose group was observed in one piglet, and it was not as severe as it was in the high-dose group. Lateral recumbency was not observed in any piglet throughout the study. Loss of vitality and appetite and troubles in respiratory systems were also

Table 1. Clinical score and body weight of the piglets with a high or a low dose of STEC ${ }^{\text {a) }}$

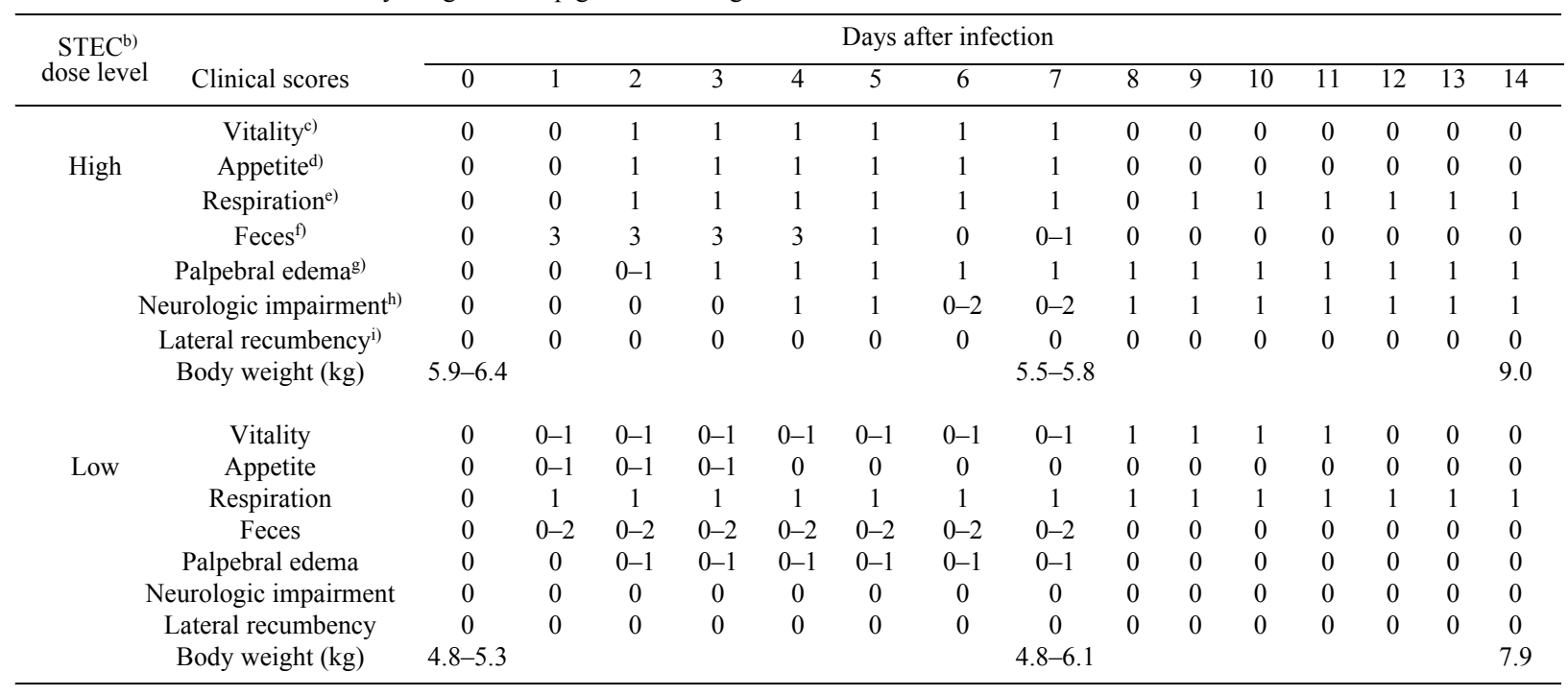

a) $\mathrm{n}=2$ (from $\mathrm{d} 0$ to $\mathrm{d} 7) ; \mathrm{n}=1$ (from $\mathrm{d} 8$ to $\mathrm{d} 14)$.

b) High, $1.0 \times 10^{10} \mathrm{CFU} /$ head/day; Low, $3.9 \times 10^{8} \mathrm{CFU} /$ head/day.

c) Vitality score: 0 , good; 1 , loose; 2 , bad.

d) Appetite score: 0, good; 1, slightly poor; 2, poor.

e) Respiratory score: 0 , normal; 1 , slightly quick; 2 , quick.

f) Fecal score: 0, normal; 1, loose stool; 2, moderate diarrhea; 3, severe diarrhea.

g) Edemal score in palpebra: 0, normal; 1, mild; 2, moderate; 3, severe.

h) Neurologic impairment score: 0 , not impairment; 1 , slightly; 2, moderate; 3 , severe.

i) Lateral recumbency was not observed in all animals throughout this experiment. 
Table 2. STEC numbers in rectal swabs of the piglets with a high or a low dose of STEC ${ }^{\text {a) }}$

\begin{tabular}{ccc}
\hline STEC dose level & Sampling day & STEC (CFU/swab) \\
\hline High & $\mathrm{d} 0$ & N.D. ${ }^{\mathrm{b}}$ \\
& $\mathrm{d} 1$ & $10^{6}-10^{8}$ \\
& $\mathrm{~d} 7$ & $10^{3}-10^{6}$ \\
Low & $\mathrm{d} 14$ & N.D. \\
& $\mathrm{d} 0$ & N.D. \\
& $\mathrm{d} 1$ & $10^{5}-10^{7}$ \\
& $\mathrm{~d} 7$ & $10^{3}-10^{6}$ \\
& $\mathrm{~d} 14$ & N.D. \\
\hline
\end{tabular}

a) $n=2(d 0, d 1$ and $d 7) ; n=1(d 14)$.

b) N.D.: STEC was not detected (less than $1.0 \times 10^{2} \mathrm{CFU} /$ swab).

Table 3. Numbers of STECs in the intestinal content of piglets with a high or a low dose of STEC ${ }^{\text {a) }}$

\begin{tabular}{ccc}
\hline STEC dose level & Intestine & STEC (CFU/swab) \\
\hline High & Jejunum & N.D. $-10^{2 \mathrm{~b})}$ \\
& Ileum & $10^{2}-10^{5}$ \\
& Cecum & $10^{3}-10^{5}$ \\
Low & Jejunum & N.D. $-10^{4}$ \\
& Ileum & $10^{4}-10^{7}$ \\
& Cecum & $10^{2}-10^{6}$
\end{tabular}

a) $n=2$; One pig was euthanized at $d 7$, and another pig was done at $\mathrm{d} 14$.

b) N.D.: STEC was not detected (less than $1.0 \times 10^{2}$ CFU/swab).

observed in the high-dose group. These symptoms were also observed in the low-dose group, but they were relatively mild. Severe diarrhea developed in the high-dose group from $\mathrm{d} 1$ to $\mathrm{d} 4$. In contrast to the high-dose group, only moderate diarrhea was observed in one piglet in the low-dose group. Body weight decreased in the high-dose group during the experiment, whereas it remained stable or increased slightly in the low-dose group. STEC was not detected $(<100 \mathrm{CFU} / \mathrm{swab})$ in the rectal swabs of any piglet on $\mathrm{d} 0$ (before infection) (Table 2). On $\mathrm{d} 1$ and $\mathrm{d} 7$, STEC was detected $\left(10^{3}\right.$ to $\left.10^{8} \mathrm{CFU} / \mathrm{swab}\right)$ in the rectal swabs of all piglets. However, STEC was not detected on d14. STEC was detected in the ileum and cecum of all piglets (Table 3). High-dose group had a tubercle in the lung (Fig. 1A) or pus in the thoracic cavity, and STEC was also detected in them.

The small intestinal tissues were severely damaged by STEC infection (Table 4). The mid-segment of the ileum showed atrophy, and loss of the villi in all piglets (Fig. 2A and C). Mucosal erosion was observed in three piglets (Fig. 2D). Edematous lamina propria with severe infiltration of lymphocytes and eosinophils was observed in the ileum of all piglets (Fig. 2A, B, C and E). Such infiltration was more serious at the end-segment of the ileum than that in the midsegment. Mucosal erosion, atrophy, and loss of the villi were observed in all piglets. Severe edema with swollen lymphoid follicles was observed in the lamina propria accompanied with the infiltration of lymphocytes and eosinophils. Unlike the ileum, the large intestine, such as the mid-segment of the gyri centripetales, was only mildly damaged by the STEC infection. Mucosal atrophy and erosion were not clear. Focal edema with slight lymphocyte infiltration was observed in the lamina propria of the gyri centripetales of all piglets. Abnormal eosinophil infiltration was not seen in the gyri centripetales. Only one piglet had swollen lymphoid follicles in the gyri centripetales (high-dose group). The lung was severely abnormal in a piglet in the high-dose group, including a tubercle with interstitial pneumonia, abscesses and congestion (Fig. 1A and B). The kidney showed focal or slight abnormalities, including a thrombus and congestion. This piglet had a swollen lymph node at the mesentery, hilus of the spleen, and inguinal region. The inguinal lymph node had a hemosiderin adhesion in the sinus subcapsularis. The lung of another piglet in the high-dose group was moderately abnormal, including pleura adhesion, congestion, and interstitial pneumonia. This piglet had focal or slight abnormalities of the kidneys (Fig. 1C), including a thrombus, and a hemorrhaged liver. This piglet also contracted pleuritis infiltrated by a large number of granulocytes in the pleura. The low-dose group had focal or slight abnormalities of the lung, including interstitial pneumonia. And, the kidney showed focal and/or slight abnormality, including a thrombus.

In previous experimental infection trials in which STEC was introduced into the stomach with a gelatin capsule or catheter, no significant clinical symptoms of ED were

Table 4. Pathological scores of the intestinal tissues of piglets with a high or a low dose of STEC ${ }^{\text {a) }}$

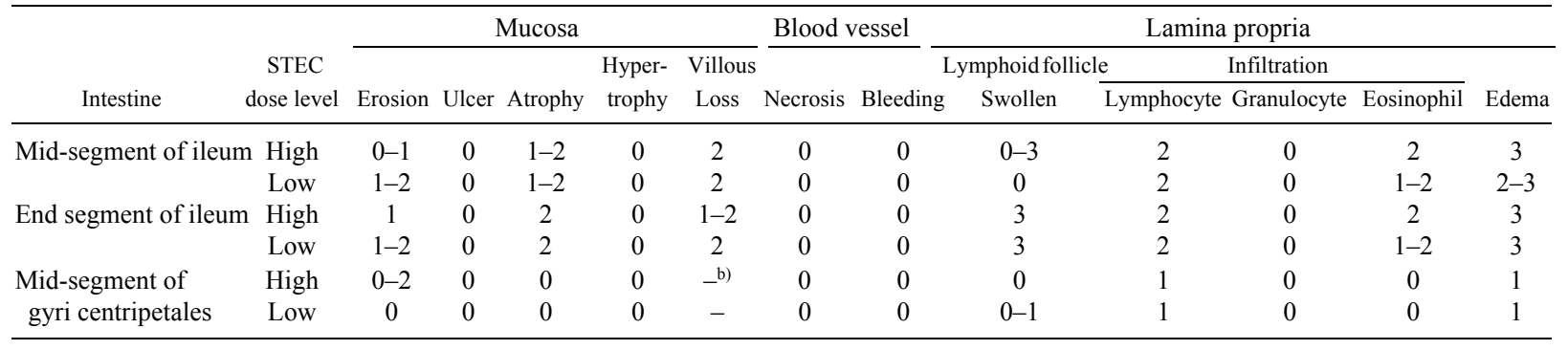

a) Pathologic score: 0 , normal; 1 , slightly abnormal or locally abnormal; 2 , moderately abnormal; 3 , severely abnormal.

$\mathrm{n}=2$; One pig was euthanized at $\mathrm{d} 7$, and another pig was done at $\mathrm{d} 14$.

b) Villi were not observed in the large intestine. 

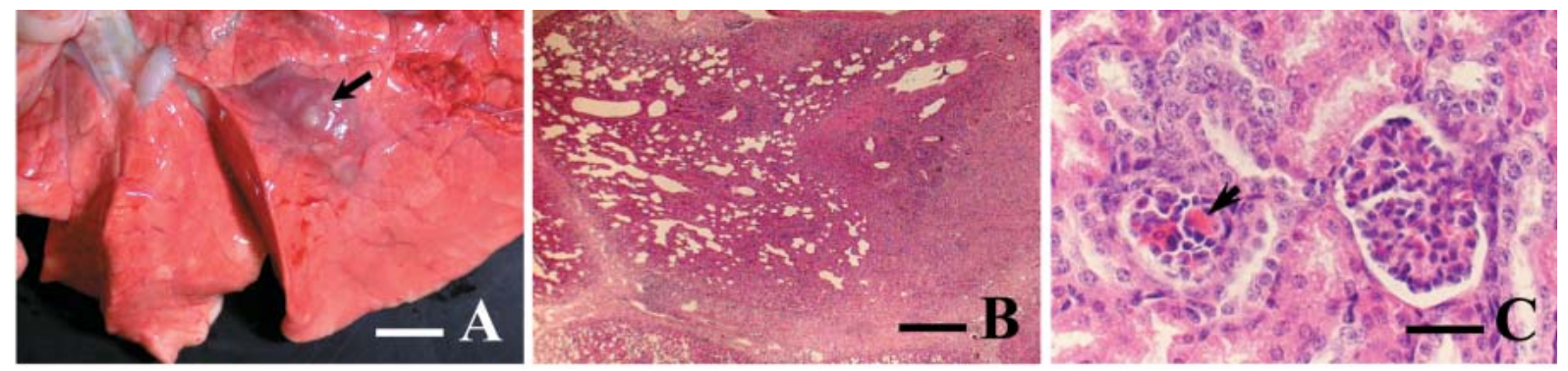

Fig. 1. Photographs of the abnormal organs of a high-dose STEC-infected piglet. A, a tubercle on a lung (a black arrow; bar represents $10 \mathrm{~mm}$ ); B, a light micrograph of a tubercle of photo A. HE stain, (bar represents $500 \mu \mathrm{m}$ ). Almost all lung tissue was afflicted by the interstitial pneumonia. Disappearance of alveoli was remarkable; C, a photomicrograph of a kidney. HE stain, (bar represents $60 \mu \mathrm{m}$ ). An arrowhead indicates a thrombus in a glomerulus.
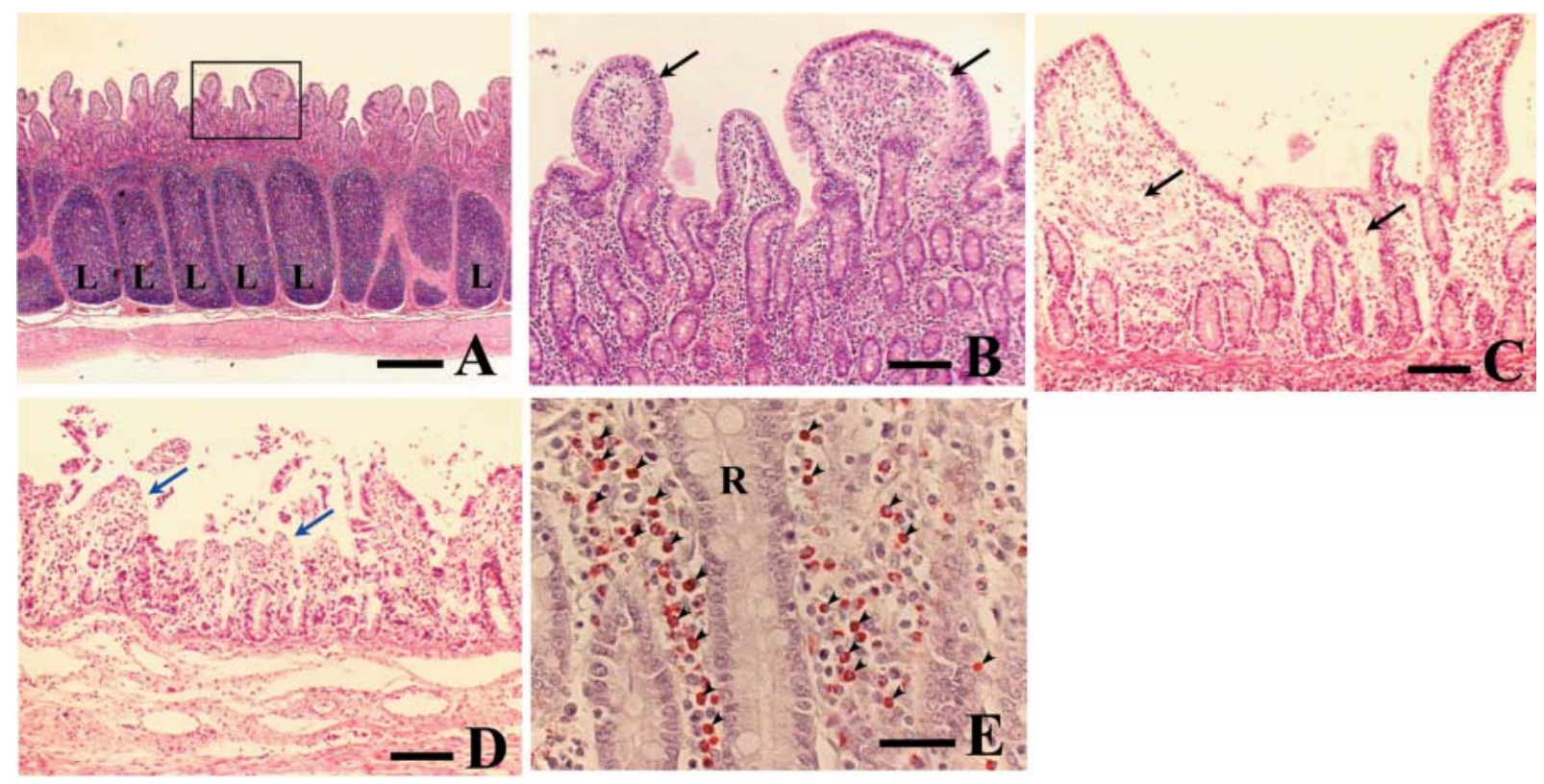

Fig. 2. Photomicrographs of the thin sections of the ileum of a high-dose STEC-infected piglet. A, Edema and swollen lymphoid follicles (L) were observed in the lamina propria. Atrophy and loss of villi were also remarkable. HE stain, (bar represents $500 \mu \mathrm{m}$ ); B, Photo B is a high magnification of photo A (Inset area). Black arrows indicate edematous villi. HE stain, (bar represents $100 \mu \mathrm{m}$ ); $\mathrm{C}, \mathrm{Severe}$ edema was observed in the lamina propria. Black arrows indicate mucosal edema. Atrophy and loss of villi were also remarkable. HE stain, (bar represents $100 \mu \mathrm{m}$ ); D, Mucosal erosion was observed (Blue arrows). HE stain, (bar represents $100 \mu \mathrm{m}$ ); E, Large numbers of eosinophils infiltrated the lamina propria (arrowheads). "R" represents a crypt in the mucosa. Luna stain, (bar represents $60 \mu \mathrm{m}$ ).

observed $[5,7,14]$. With these methods, STEC was poured into the stomach of piglets, where a low $\mathrm{pH}$ is generally assured and large numbers $\left(10^{7} \mathrm{cell} / \mathrm{g}\right)$ of lactic acid bacteria, such as lactobacilli and streptococci, exist [15]. Such low $\mathrm{pH}$ is obviously bactericidal. Lactic acid bacteria and lactobacilli, in particular, are known to eliminate $E$. coli and Salmonella from the digestive tract [6]. Furthermore, pigs keep the ingested food in the stomach for relatively long periods ( 3 to $13 \mathrm{hr}$ ) [24]. Therefore, a large proportion of STEC introduced by these methods should have been killed within the stomach, and therefore few bacteria colonized the small intestine in the above-mentioned previous infection trials. Passing through the stomach barrier without loss of STEC is therefore crucial to establish the infection model.
In this experiment, we used capsules resistant to gastric digestion. Since this capsule is labile to intestinal digestion, it delivered STEC intact to the small intestine. The level of STEC in the rectal swabs ranged from $10^{5}$ to $10^{8} \mathrm{CFU} / \mathrm{swab}$ at d1, which seems sufficient to induce ED in piglets.

STEC infection seriously damaged the small intestine of the piglets. Edematous lamina propria with swollen lymphoid follicles was observed in this study. Large numbers of eosinophils and lymphocytes infiltrated the abnormal portion of the lamina propria. However, other granulocytes, mainly neutrophils, were not observed in these areas. Infiltration of eosinophils was shown by previous reports in the STEC infected pigs $[13,17]$. Thus, their infiltration is a common pathological characteristic of the STEC-infected 
piglets. In the human in vivo study, STEC infection induces neutrophil infiltration in the colonic lamina propria and crypts [9]. STEC infects the small intestine in the case of pigs, but it infects the large intestine in human cases. Eosinophil infiltration is as well-known a response as is the allergic inflammation in food allergy and asthma, which may contribute to tissue damage and connective tissue remodeling $[1,16]$. Eosinophils have several properties that contribute to bactericidal activity in tissue, such as cytotoxic protein production and reactive oxygen species production [18]. Accordingly, experimental infection of STEC in weaned piglets triggers local allergic reactions in the small intestine as suggested by the earlier report [17]. Stx 2e is transported to sensitive tissues, such as the brain and kidney, with the vascular system causing vascular necrosis and edema in those organs. Neurologic symptoms are also evident and often kill the patients $[12,17]$. STEC was detected in the lung or thoracic cavity of piglets with a high-dose infection by the bacteriological method. This is distinct from human STEC, which is generally believed to be a noninvasive bacterium [10]. Therefore, the etiology of STEC diseases of human $[9,10]$ and porcine $[2,12,17]$ origins might be different. In conclusion, the experimental infection of porcine STEC was successful by the use of the capsule resistant to gastric digestion. This method is applicable to other porcine enteropathogens, i.e., enterotoxigenic $E$. coli and Brachyspira hyodysenteriae.

ACKNOWLEDGEMENTS. The authors thank Ms. J. Hayashi, Mr. M. Nakamoto, Mr. K. Ohya, Mr. A. Tomita, Mr. K. Utsumi, and Ms. N. Wada in the KYODOKEN Institute, Mr. M. Nishikawa in the Kyoto Institute of Nutrition and Pathology, and Ms. M. Tanimura in the Japan Cytology Research for their technical assistance.

\section{REFERENCES}

1. Arima, M. 2004. Igakuno Ayumi 211: 701-706 (in Japanese).

2. Bertschinger, H. U. and Gyles, C. L. 1994. In: Escherichia coli in Domestic Animals (Gyles, C. L. ed.), CAB International, Wallingford.

3. Blanco, M., Blanco, J. E., Gonzalez, E. A., Mora, A., Jansen, W., Gomes, T. A. T., Zerbini, L. F., Yano, T., Pestana de Castro, A. F. and Blanco, J. 1997. J. Clin. Microbiol. 35: 29582963.

4. Bosworth, B. T., Dean-Nystrom, E. A., Casey, T. A. and Neibergs, H. L. 1998. Clin. Diagn. Lab. Immunol. 5: 299-302.
5. Bosworth, B. T., Samuel, J. E., Moon, H. W., O’Brien, A. D., Gordon, V. M. and Whipp, S. C. 1996. Infect. Immun. 64: 5560 .

6. Chateau, N., Castellanos, I. and Deschamps, A. M. 1993. J. Appl. Bacteriol. 74: 36-40.

7. Cornick, N. A., Matise, I., Samuel, J. E., Bosworth, B. T. and Moon, H. W. 2000. J. Infect. Dis. 181: 242-251.

8. Fukuyama, M., Furuhata, K., Oonaka, K., Yakiwara, R., Koizumi, T., Hara, M., Dogasaki, C. and Watanabe, T. 2003. Kansensyogaku-Zasshi 77: 1032-1039 (in Japanese with English summary).

9. Griffin, P. M., Olmstead, L. C. and Petras, R. E. 1990. Gastroenterology 99: 142-149.

10. Hurley, B. P., Thorpe, C. M. and Acheson, W. K. 2001. Infect. Immun. 69: 6148-6155.

11. Johnson, W. M., Pollard, D. R., Lior H., Tyler, S. D. and Rozee, K. R. 1990. J. Clin. Microbiol. 28: 2351-2353.

12. Kashiwazaki, M. 1987. pp. 395-401. In: Hyology and Hyoiatrics, 3rd ed. (Kumagai, T., Azuma, R., Kashiwazaki, M., Kawata, K., Shimizu, Y., Namioka, S., Yoshimoto, T., and Watanabe, S. eds.), Kindaisyuppan, Tokyo (in Japanese).

13. Kurz, H. J., Bergeland, M. E. and Barnes, D. M. 1969. Am. J. Vet. Res. 30: 791-806.

14. Matise, I., Cornick, N. A., Samuel, J. E. and Moon, H. W. 2003. Infect. Immun. 71: 5194-5201.

15. Mitsuoka, T. 1987. pp. 107-119. In: Hyology and Hyoiatrics, 3rd ed. (Kumagai, T., Azuma, R., Kashiwazaki, M., Kawara, K., Shimizu, Y., Namioka, S., Yoshimoto, T. and Watanabe, S. eds.), Kindaisyuppan, Tokyo (in Japanese).

16. Nowak-Wegrzyn, A. 2003. Pediatrics 111: 1672-1680.

17. Ohshima, K-I. and Miura, S. 1961. Jpn. J. Vet. Sci. 23: 111121.

18. Persson, T., Andersson, P., Bodelsson, M., Laurell, M., Malm, J. and Egesten, A. 2001. Infect. Immun. 69: 3591-3596.

19. Tsukahara, T. and Ushida, K. 2001. J. Vet. Med. Sci. 63: 13511354.

20. Tsukahara, T., Iwasaki, Y., Nakayama, K. and Ushida, K. 2003. J. Nutr. Sci. Vitaminol. 49: 414-421.

21. Tsukahara, T., Iwasaki, Y., Nakayama, K. and Ushida, K. 2003. J. Vet. Med. Sci. 65: 301-306.

22. Uemura, R., Sueyoshi, M., Nagayoshi, M. and Nagatomo, H. 2003. Microbiol. Immunol. 47: 57-61.

23. Uemura, R., Sueyoshi, M., Taura, Y. and Nagatomo, H. 2004. J. Vet. Med. Sci. 66: 899-903.

24. Warner, A. C. I. 1981. Nutr. Abstr. Rev. 51B: 789-821.

25. Watanabe, K. and Yoshiura, N. 2001. J. Vet. Epidemiol. 5: 2529 (in Japanese with English summary).

26. Yuji, H., Tsuji, A. and Sueyoshi, M. 2000. J. Vet. Clin. 47: 423-428 (in Japanese with English summary). 